\title{
Intestinal Ischemia as a Complication of COVID-19
}

\author{
Lamis K. Abufool Labib S. Al-Ozaibi \\ General Surgery Department, Rashid Hospital, Dubai Health Authority, Dubai, United Arab Emirates
}

\section{Keywords}

COVID-19 · SARS-CoV-2 · Mesenteric ischemia · Thrombosis · Endothelial activation

\begin{abstract}
Background: Extrapulmonary complications of COVID-19 including acute mesenteric ischemia are being increasingly reported and are a reason of poorer clinical outcome and increased inpatient mortality rate. Case: Here, we present a case of a young obese male who developed acute superior mesenteric artery thrombosis as a result of COVID-19. Discussion: Some direct causes of mesenteric thrombosis in COVID-19 patients have been hypothesized. Furthermore, immobilization, endothelial injury, and increased prothrombotic factor production are proven causes of thromboembolism in COVID-19. Conclusion: Early detection and management of acute mesenteric ischemia in COVID-19 cases is crucial to improve the prognosis. @ 2021 The Author(s).

Published by S. Karger AG, Basel
\end{abstract}

\section{Introduction}

With emerging data from increased research pointed toward the SARS-CoV-2 pandemic, it is now well known that the disease does not affect the respiratory system alone. Shortly after the rise of the COVID-19 virus, gas- trointestinal symptoms started to be described and were reported to be self-limiting in the majority of cases [1]. COVID-19-related coagulopathies are becoming increasingly common, and patients are rapidly presenting with severe venous and arterial thromboembolic complications in addition to the respiratory complications of the disease. The pathogenesis implicates Virchow's triad and is most likely due to endothelial injury, immobilization, and rising circulatory prothrombotic factors despite generous anticoagulation and protocolized care [2]. In this case report, we present an unfortunate thromboembolic effect of COVID-19.

\section{Case Presentation}

A 38-year-old obese (BMI $35.93 \mathrm{~kg} / \mathrm{m}^{2}$ ) male patient with no known medical or surgical history presented to our hospital with fever, dry cough, and shortness of breath of 2-day duration and a positive COVID-19 PCR test. The patient had not received any immunization against COVID-19. On examination, the patient was vitally stable. He had a normal chest and cardiovascular examination with no abdominal tenderness. On chest X-ray, a patch of pneumonic consolidation was noted involving the left lower lobe of the lung.

Four days later, the patient developed respiratory distress. He was tachycardiac with a pulse of 100 beats per minute, tachypneic with a respiratory rate of 36 breaths per minute, hypotensive with a blood pressure of $90 / 54 \mathrm{~mm} \mathrm{Hg}$, and hypoxic with an oxygen saturation of $82 \%$ on a nonrebreather mask. He had bilateral crepitations on chest examination and a soft and lax abdomen. A repeat chest X-ray showed increasing bilateral patchy infiltrates.
C 2021 The Author(s).

Published by S. Karger AG, Basel

This is an Open Access article licensed under the Creative Common Attribution-NonCommercial-4.0 International License (CC BY-NC) (http://www.karger.com/Services/OpenAccessLicense), applicable to the online version of the article only. Usage and distribution for commercial purposes requires written permission.
Correspondence to:

Lamis K. Abufool, lamiskabufool@gmail.com 


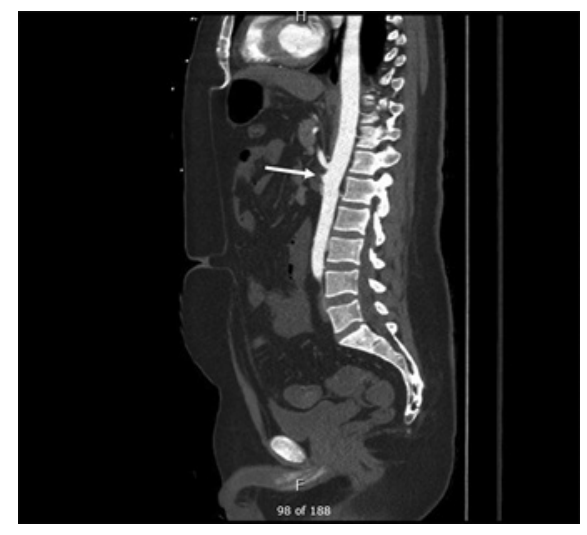

Fig. 1. Sagittal view of CT-angiogram showing hypodense filling defect of the superior mesenteric artery starting at its origin and involving its whole length (white arrow).

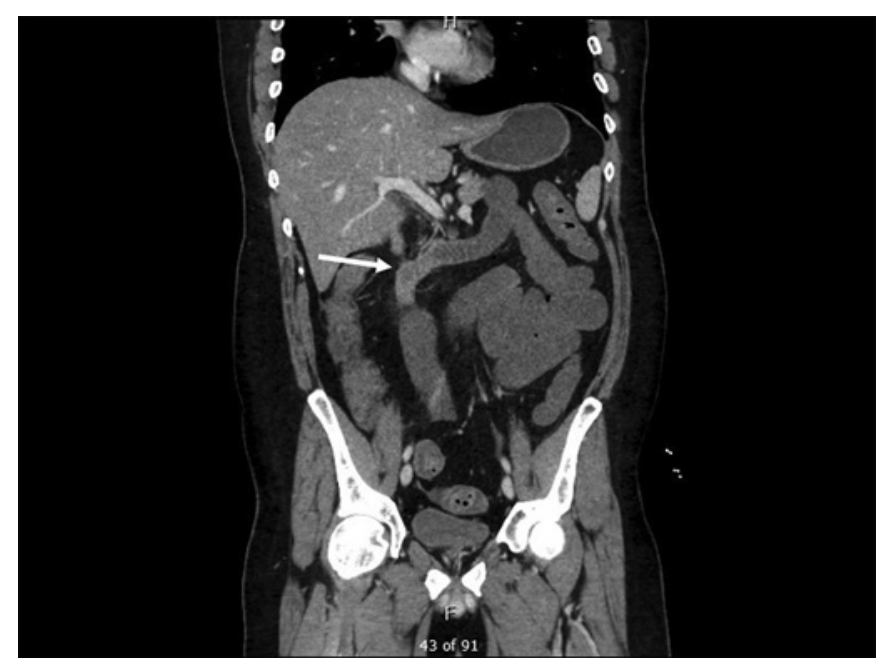

Fig. 2. Coronal view of CT-angiogram showing a single enhanced jejunal loop (white arrow). In comparison, all the other bowel loops show dilation and hypoenhancement denoting ischemia. There is stranding of the mesenteric fat planes representing mesenteric congestion.

He was labeled as a case of severe COVID-19 pneumonia which was worsening clinically and radiologically. He was started on a therapeutic dose of enoxaparin of $80 \mathrm{mg}$ subcutaneously twice a day.

On the 5th day of admission, the patient developed severe persistent abdominal pain which was not relieved by analgesia or proton pump inhibitors. He was diaphoretic and had one bout of vomiting. The patient was vitally stable and had a GCS of 15/15. Per abdominal examination showed a lax abdomen with mild tenderness in the epigastrium on palpation. His D-dimer had risen to $12.91 \mu \mathrm{g} / \mathrm{mL}$. The pain persisted, and the patient underwent an urgent abdominal CT-angiogram with findings of hypodense filling defect of the superior mesenteric artery and vein denoting arterial and venous thrombosis associated with ischemic changes of the small bowel loops (shown in Fig. 1, 2).

Intestinal Ischemia as a Complication of COVID-19
He was taken for an exploratory laparotomy. Operative findings were of ischemic nonviable bowel from the duodenojejunal flexure to the mid transverse colon. The ischemic bowel was resected, and the patient was transferred to the surgical intensive care unit postoperatively.

Postoperatively, the patient developed sepsis and multiorgan failure. He developed pulseless ventricular tachycardia and was resuscitated according to Advanced Cardiovascular Life Support guidelines. Return of spontaneous circulation was unfortunately not achieved, and the patient passed away 9 days after admission on postoperative day 3 .

\section{Discussion}

Respiratory, cardiovascular, neurological, gastrointestinal, and vascular complications have all been reported with COVID-19 infections whether as presenting symptoms or as complications of the disease. Coagulopathy and increased D-dimer levels on admission have been associated with a poorer clinical course and an increased inpatient mortality rate [3].

SARS-CoV-2 infects the host cells using angiotensin converting enzyme 2 which is a zinc-dependent metallopeptidase expressed by the cells of many organs including the lung, heart, intestine, and kidney. In other words, ACE-2 acts as a functional receptor of the virus. This means that endothelial dysfunction leading to hypercoagulability exists specifically in the bowel and is a direct cause of mesenteric thrombosis in COVID-19 patients.

ACE-2 is also expressed by endothelial cells causing endothelial cell involvement across vascular beds of different organs. Consequently, this recruitment of immune cells can cause endothelial dysfunction with apoptosis. This may explain the resultant organ ischemia as endothelial dysfunction is one of the principal determinants of microvascular dysfunction leading to vasoconstriction [4]. In addition, levels of D-dimer, fibrinogen, Factor VIIIc, and von Willebrand factor are higher in COVID-19 patients when compared to their levels in severely septic COVID-19-negative patients admitted to the ICU [5]. Immobilization leading to stasis of blood also plays a significant role in COVID-19-associated hypercoagulability. Moreover, edema and the cytokine storm related to the high inflammatory state leading to coagulation and fibrinolysis activation are also predisposing factors to organ ischemia $[6,7]$.

An interesting study done in August 2020 showed the high prevalence of early asymptomatic venous thromboembolism in patients who were receiving anticoagulation. The physicians performed a complete Doppler ul- 
trasound on patients 4 days after their admission regardless of their D-dimer levels. The study resulted in $19 \%$ of patients having asymptomatic deep venous thrombosis (8 out of 42 patients). Seven of those 8 patients had distal thrombosis and 4 of them had bilateral DVT. The study denotes the prevalence of DVT and overall worse prognosis in the older age group of their study [8]. Asymptomatic DVT may be a reason of unexplained poorer outcome in these patients.

Acute mesenteric ischemia is increasingly being reported as an extrapulmonary complication of COVID-19 infections. Because of its oblique angle in relation to the aorta, the superior mesenteric artery is particularly susceptible to emboli that can lodge. Acute mesenteric ischemia complicating severe COVID-19 infections may present with abdominal pain out of proportion to physical examination, nausea, vomiting, diarrhea, constipation, eructation, abdominal distention, fever, and/or sepsis [9, 10]. Laboratory investigations may show metabolic acidosis with elevated anion gap as well as elevating levels of lactate and D-dimer. These tests, however, are nonspecific as they are usually elevated in patients with COVID-19. The mainstay of diagnosis is early CT-angiography which holds a crucial role in the timely detection and management of this life-threatening complication.

\section{Conclusion}

COVID-19 can present in countless shapes and forms. The patients can present with mild or severe upper respiratory tract infection, deep venous thrombosis, pulmonary embolism, cerebrovascular accident, bowel ischemia, or can even be asymptomatic. Some risk factors have been implicated in the severity of the disease, but the prognosis can never be predicted. Anticoagulation therapy introduced in the early disease could prevent coagulopathy and endotheliopathy leading to better outcomes.
However, even with protocolized care and early treatment including prophylactic or therapeutic anticoagulation, patients can still develop severe and fatal forms of the disease.

\section{Statement of Ethics}

As the patient passed away and the next kin was not reachable, the Dubai Scientific Research Ethics Committee Approved a waiver of consent with the following reference number: DSRECGL05-20201 dated September 6, 2021, in accordance with the below statement: "The committee has accepted and approved the request for waiver of consent for following reasons: (a) the patient described in the case report passed away, and the next of kin was unreachable. (b) The patient's privacy was protected throughout the entire report. (c) For the importance of the case for science, a written informed consent was obtained from the patient's most responsible physician for the publication of this case and any accompanying images."

\section{Conflict of Interest Statement}

The authors have no conflicts of interest to disclose.

\section{Funding Sources}

No funding was received.

\section{Author Contributions}

L.K.A. contributed to data collection and writing the manuscript. L.S.A.-O. contributed to critical review and final approval.

\section{Data Availability Statement}

All data generated or analyzed during this case report are included in this article. Further enquiries can be directed to the corresponding author.

\section{References}

1 Jin X, Lian JS, Hu JH, Gao J, Zheng L, Zhang $\mathrm{YM}$, et al. Epidemiological, clinical and virological characteristics of 74 cases of coronavirus-infected disease 2019 (COVID-19) with gastrointestinal symptoms. Gut. 2020;69(6): 1002-9.

2 Llitjos JF, Leclerc M, Chochois C, Monsallier JM, Ramakers M, Auvray M, et al. High incidence of venous thromboembolic events in anticoagulated severe COVID-19 patients. J Thromb Haemost. 2020;18(7):1743-6.
3 Tang N, Li D, Wang X, Sun Z. Abnormal coagulation parameters are associated with poor prognosis in patients with novel coronavirus pneumonia. J Thromb Haemost. 2020;18(4): 844-7.

4 Ferrario CM, Jessup J, Chappell MC, Averill DB, Brosnihan KB, Tallant EA, et al. Effect of angiotensin-converting enzyme inhibition and angiotensin II receptor blockers on cardiac angiotensin-converting enzyme 2 . Circulation. 2005;111(20):2605-10. 
5 Ladikou EE, Sivaloganathan H, Milne KM, Arter WE, Ramasamy R, Saad R, et al. Von Willebrand factor (vWF): marker of endothelial damage and thrombotic risk in COVID-19? Clin Med. 2020;20(5):e178-82.

6 Bonetti PO, Lerman LO, Lerman A. Endothelial dysfunction: a marker of atherosclerotic risk. Arterioscler Thromb Vasc Biol. 2003; 23(2):168-75.
7 Panigada M, Bottino N, Tagliabue P, Grasselli G, Novembrino C, Chantarangkul V, et al. Hypercoagulability of COVID-19 patients in intensive care unit: a report of thromboelastography findings and other parameters of hemostasis. J Thromb Haemost. 2020; 18(7):1738-42.

8 Le Jeune S, Suhl J, Benainous R, Minvielle F, Purser C, Foudi F, et al. High prevalence of early asymptomatic venous thromboembolism in anticoagulated COVID-19 patients hospitalized in general wards. J Thromb Thrombolysis. 2021;51(3):637-41.
9 Parry AH, Wani AH, Yaseen M. Acute mesenteric ischemia in severe Coronavirus-19 (COVID-19): possible mechanisms and diagnostic pathway. Acad Radiol. 2020;27(8): 1190.

10 Singh B, Kaur P. COVID-19 and acute mesenteric ischemia: a review of literature. $\mathrm{He}$ matol Transfus Cell Ther. 2021 Jan-Mar; $43(1): 112-6$. 